SESSION 2

\title{
OBSERVATIONS OF THE CRAB PULSAR
}




\title{
2.1 RADIO OBSERVATIONS OF THE CRAB NEBULA PULSAR
}

\author{
F. D. DRAKE \\ Cornell-Sydney University Astronomy Center, Cornell University, Ithaca, N.Y., U.S.A.
}

\begin{abstract}
The radio properties of the Crab Nebula pulsar are reviewed. The pulsar lies at the centre of the Crab Nebula and has a period of $33 \mathrm{msec}$. Its increase in period with time releases an amount of energy which is equal in magnitude to the total radiated power. Instabilities in the period of the Crab pulsar have been discovered with timescales ranging from days to months. The length of the pulse increases at longer wavelengths due apparently to multipath propagation effects. A characteristic of the Crab pulsar is the great intensity of the occasional pulse.
\end{abstract}

The Crab Nebula pulsar is one of the most important objects in the history of astronomy. It alone has given us convincing evidence that the pulsars are rapidly rotating neutron stars in which the source of emissions we see is their rotational kinetic energy. These objects apparently convert this energy into electromagnetic radiation through the intermediary of a very intense magnetic field. With the Crab Nebula, its pulsar has proved the missing link so long sought to explain some of the most important features of the nebula, particularly its source of energy and the unique point source of low frequency radio emission observed in the centre of the nebula. This pulsar, of course, has led to the only optical and X-ray observations of any pulsar.

The existence of the pulsar became known in the latter part of 1968. Staelin and Reifenstein (1968) at the National Radio Astronomy Observatory observed brief pulses from the vicinity of the Crab Nebula which, although not apparently periodic, showed the famous dispersion effect characteristic of pulsar radiation. In fact, two dispersion measures were present which led Staelin and Reifenstein to hypothesize that there were either two pulsars or some new type of pulse emitting object in the vicinity of the Crab Nebula. Very shortly thereafter Comella et al. (1969) at the Arecibo Observatory showed that indeed the sources of the radiations were pulsars, one of which is now the famous Crab Nebula pulsar NP 0532 with its remarkably short period of about $33 \mathrm{msec}$. The other pulsar, NP 0527, is located nearly a degree and a half from the Crab Nebula and has the longest period of any known pulsar, $3.75 \mathrm{sec}$. The Arecibo data enabled Cocke et al. (1969) to detect optical pulsed radiation from the well known south-preceding member of the central pair of stars in the nebula. Thus the location and period of the pulsar were well established.

Shortly after the discovery of the radio pulsar, Richards and Comella (1969) at Arecibo detected in it the first known change in pulsar period. In this case, as in all subsequent cases, it was found that the period was increasing with time. In the case of the Crab Nebula pulsar, this increase in period is about $36 \mathrm{nsec}$ per day. Though very small, this rate in one solar mass object of some ten kilometers radius rotating 30 times a second represents a rotational kinetic energy loss of some $10^{38}$ erg per sec. Thus, the pulsar is releasing energy at a rate equivalent to $10^{5}$ solar luminosities. This 
power output is remarkable not only for its magnitude but also because it is equal to the total radiated power of the Crab Nebula at all wavelengths. The origin of this power has long been a major puzzle; at last the pulsar seemed to offer a ready explanation of the source of energy. As was noted soon thereafter by Gold (1969), the fact that so much of the rotational energy of the pulsar was released in the form of relativistic particles suggests that pulsars may indeed be the dominant source of cosmic rays.

Radio studies of the pulsar pulse shape have shown it to be unique and remarkable. In Figure 1 we see the mean pulse shape as observed at $430 \mathrm{MHz}$ by Rankin et al. (1970). Here the pulse shape is the mean of many thousands of pulses. As can be seen, the pulse contains three strong components. There is an intense but brief 'main pulse' component lasting some $250 \mu \mathrm{sec}$ and a very similar although somewhat less intense 'interpulse' component. Preceding both of these is a broad and weak component known as the 'precursor'. The time interval between the well determined centroids of the main and interpulse components is $13.37 \pm 0.03 \mathrm{msec}$. This is the same as the time interval between the two components of the optical pulse to within $32 \mu \mathrm{sec}$ as observed by Horowitz, Papaliolios, and Carleton. The pulse timing

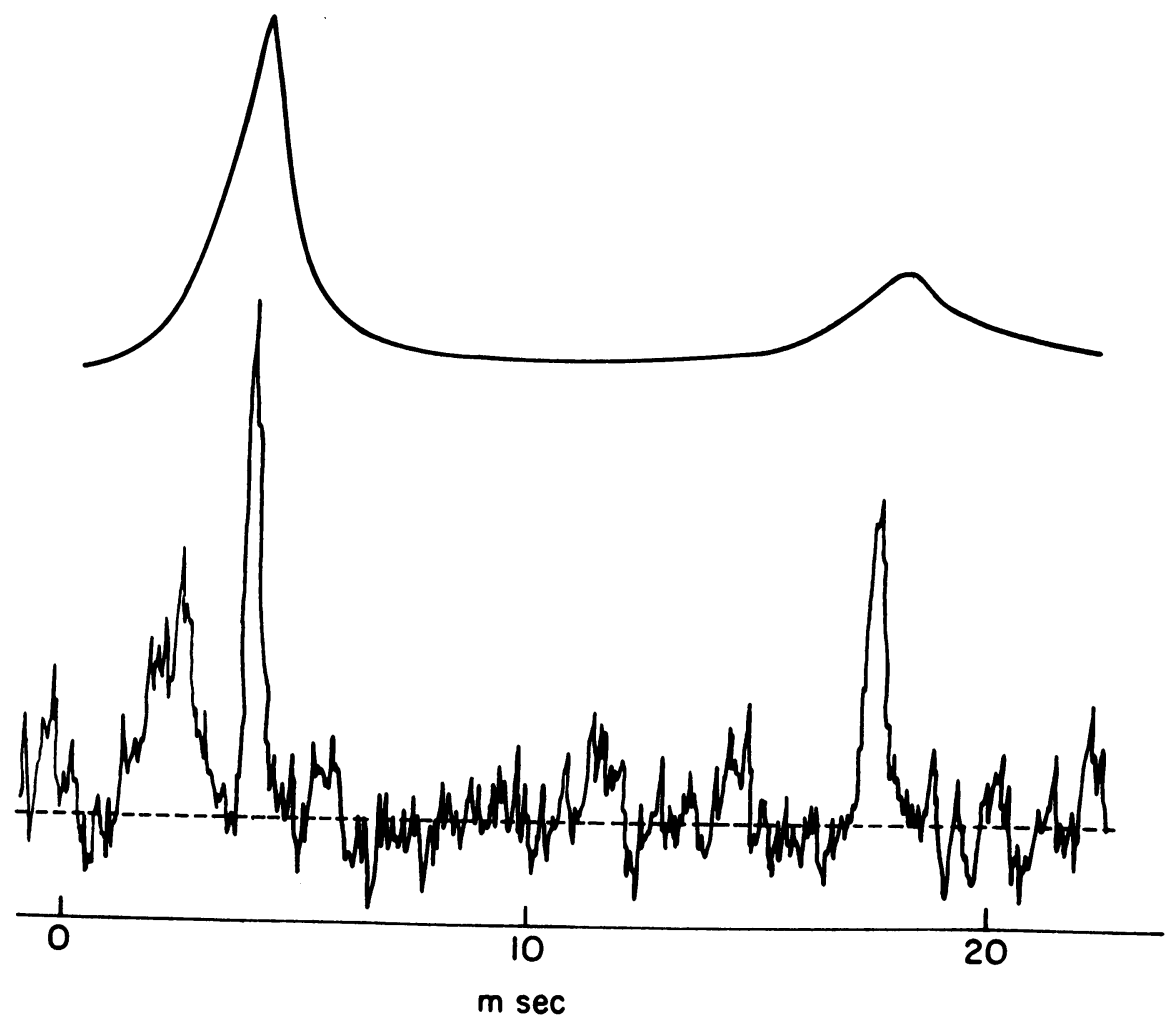

Fig. 1. Below, observed average pulse shape of NP 0532 at $430 \mathrm{MHz}$.

Above, average optical pulse shape. 
measurements have shown that the radio and optical pulses occur simultaneously after correction for dispersions to something better than $200 \mu \mathrm{sec}$. This implies that the radio and optical pulses are generated in regions that are separated by no more than $60 \mathrm{~km}$. Since this distance is very much less than the dimensions of the magnetosphere of the object, some $1000 \mathrm{~km}$, it implies strongly that the optical and radio pulses are indeed produced in the same material, although not necessarily by the same physical process.

Studies of the strengths of the various components of the pulse shape over time intervals of days have shown a small but significant relative variation of the pulse component intensities as shown in Figure 2, which is from the results of Campbell et al. (1970).

If the pulse shape is observed at many radio frequencies, it is found that major changes occur. In this respect, NP 0532 differs markedly from other pulsars. Figure 3a shows the pulse shapes measured at 74, 111, 196, 318 and $430 \mathrm{MHz}$ by Rankin et al. (1970). The very well defined and brief components seen at the highest radio frequencies become broadened as one goes to lower frequencies. The relative strength of the

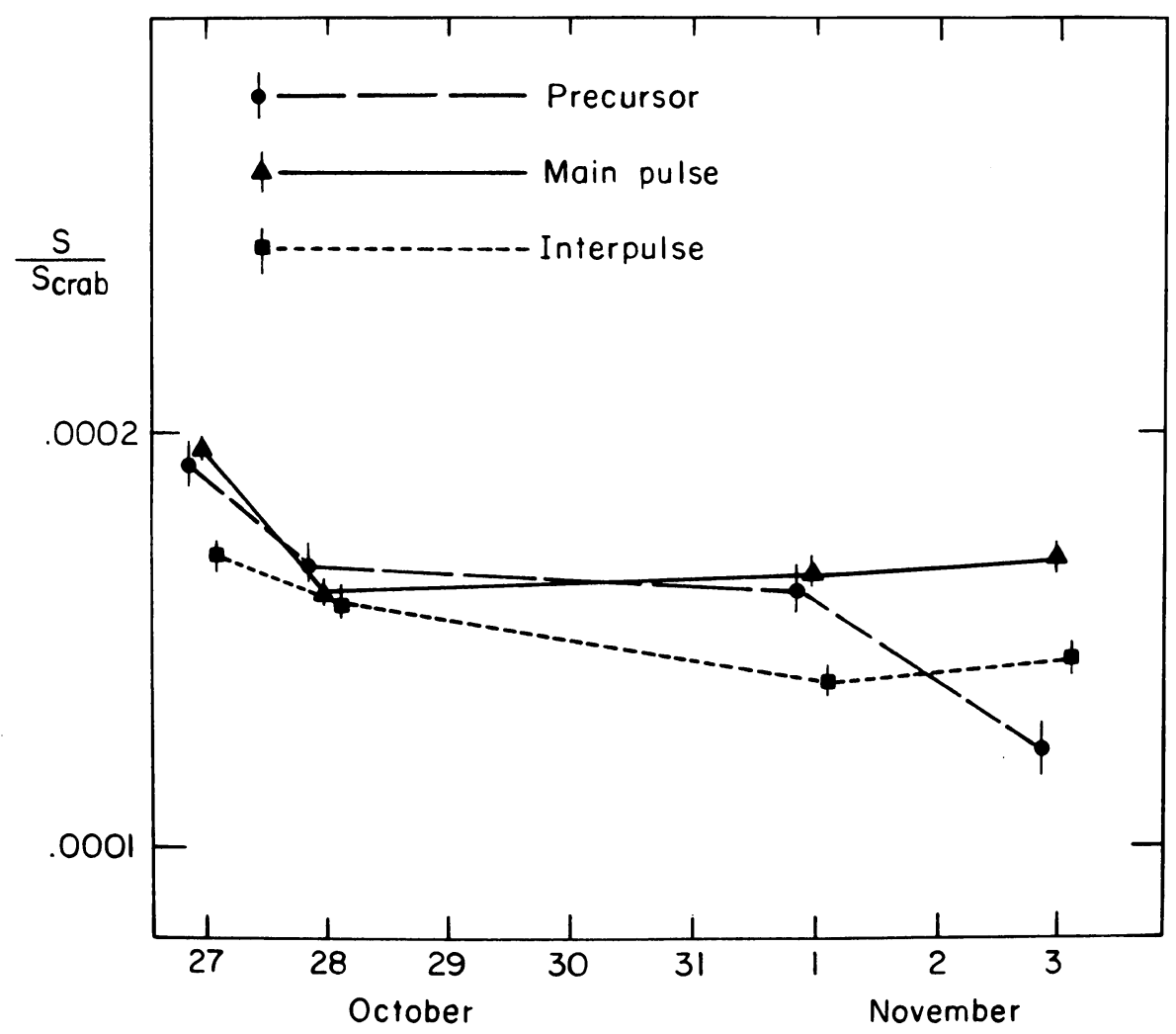

Fig. 2. Mean flux densities of three components of Crab pulsar pulse on several days in 1969. Observations were made at $430 \mathrm{MHz}$, and flux density is given in terms of the flux density of the Crab Nebula. Errors are probable errors. 


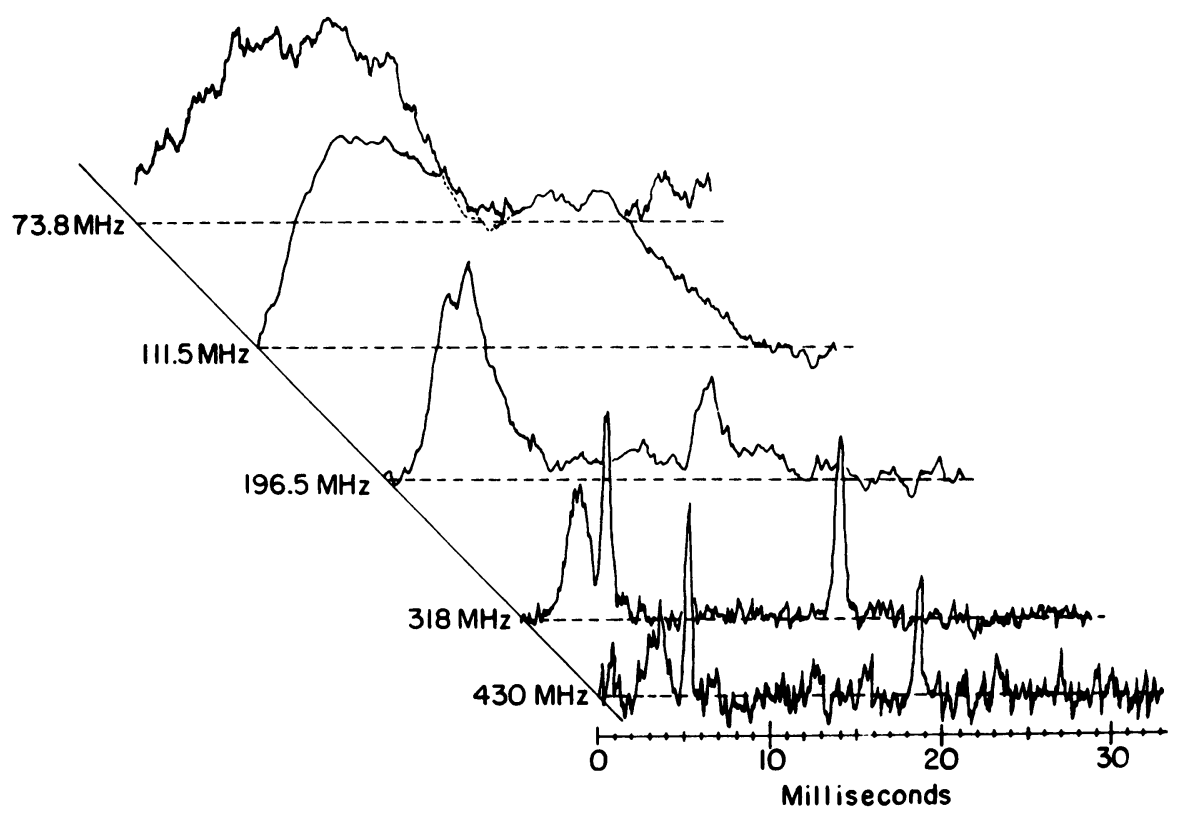

Fig. 3. Observed average pulse shape of Crab pulsar at frequencies shown. The phases of the various pulse shapes have been shifted by arbitrary amounts.

precursor increases compared to the main pulse and interpulse at the lower frequencies.

A recent observation by Heiles and Rankin at Arecibo shows that the precursor is extremely weak or perhaps unobservable at $611 \mathrm{MHz}$. The fact that the main and interpulse components can be defined well at 430, 318 and $196 \mathrm{MHz}$ permits a very accurate measurement of the dispersion measure. On 31 May 1969, the dispersion measure was $56.78 \pm 0.02$ parsec-electrons per cubic centimeter. As will be reported by Rankin, detectable and interesting changes in the dispersion measure occur.

The idea that the observed changes in pulse shape are due to multipath radio propagation in the interstellar medium has been proposed by many people, among them, Cronyn, Drake, Lang and Comella. In an attempt to study this quantitatively, Rankin et al. (1970) tried to fit various models of radio frequency dependent broadening functions to a hypothetical pulse shape to determine whether the observed pulse shapes could be reproduced. In doing this they assumed that the pulse shape observed at $430 \mathrm{MHz}$ was very nearly the intrinsic pulse shape at the pulsar. Among broadening functions they have tried are the Gaussian function, a truncated exponential, and a half Gaussian function. The function which works best is

$$
x e^{-x}(x \geqslant 0) \text { where } x=\sqrt{ } 2 \Delta t / W .
$$

This function gives the intensity that would be observed at time increment $\Delta t$ after $t=0$, if the signal at the source is a delta function at $t=0 . W=A_{0}\left(f_{0} / f\right)^{\delta}$ where $A$ and $\delta$ are to be determined from the observations. $f=$ radio frequency. Figure $3 b$ 


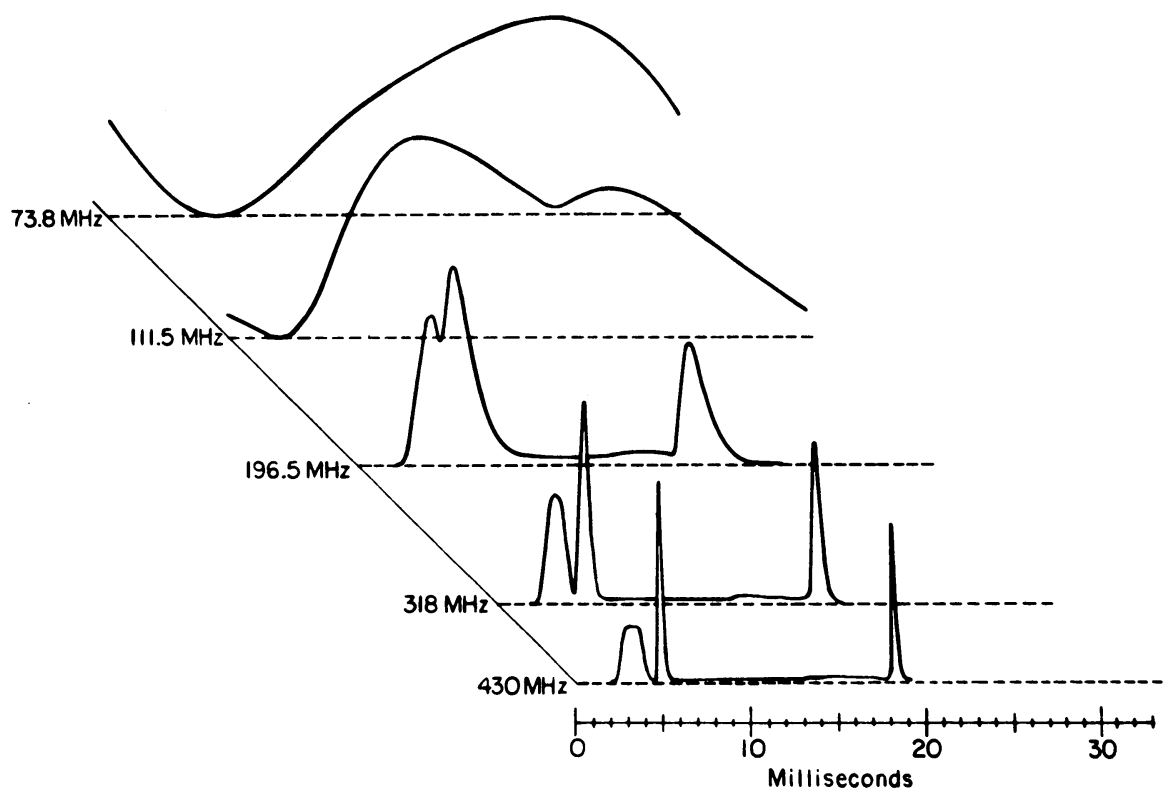

Fig. 3b. Model pulse shapes predicted from model in which the intrinsic pulse shape is defined by the average pulse shape at $430 \mathrm{MHz}$, and this pulse shape is broadened by a function $x e^{-x}$ as a result of multipath propagation effects in the interstellar medium. The broadening caused by dispersion and radiometer effects has also been included, so that these model pulse shapes are directly comparable to the pulse shapes of Figure 3.

gives the model pulse shapes found in this way when $\delta=4$ and $A_{0}=0.85$ at $f_{0}=196$ $\mathrm{MHz}$. A value $\delta=4$ gives the best fit to the data, although $3.5<\delta<4.5$ is about as good.

It is interesting that the theory of Salpeter (1969) argues that the value of $\delta$ should equal 4 in a wide range of circumstances. Thus the value observed seems to support this contention, and in the process gives weight to the hypothesis that the observed pulse broadening is due to multipath propagation in the interstellar medium. Very recently, Lang has observed the same effect, with the same mathematical dependence, in the pulse shapes of JP 1933. Additional confirmation comes from the work of Staelin and Sutton (1970) who have observed single strong pulses of NP0532 at frequencies near $100 \mathrm{MHz}$, and have found these broadened to a shape consistent with (1). There seems little doubt that gross pulse broadening at the lower radio frequencies is caused by multipath effects in the interstellar medium. The spectrum of the pulsar will be strongly affected at the lower radio frequencies as the pulse smearing leads to a conversion of pulsed radiation into continuous radiation. At a sufficiently low frequency, the pulsar will appear as a discrete continuous source and will no longer be recognized by the characteristic pulsed radiation of a pulsar.

The spectrum of the pulsar, as observed by Comella (reported in Rankin et al.) is shown in Figure 4. This is an unusual spectrum with a very steep slope on the high 
frequency side of $-2.9 \pm 0.4$. Again on the low frequency side a very steep slope occurs such that the radiation falls off so rapidly that the spectral index can not be well defined. Peak intensity occurs at a frequency of about $100 \mathrm{MHz}$. Note that this spectrum is that of the pulsed radiation alone.

Figure 4 shows the pulsed flux density predicted by the models of pulse broadening constructed by Rankin et al. In particular, the models from relation (1) are shown for $\delta$ in the range 3.5 to 4.5 , and it is seen that the spectrum follows the model predictions very precisely, with the assumption that the intrinsic spectrum at the pulsar

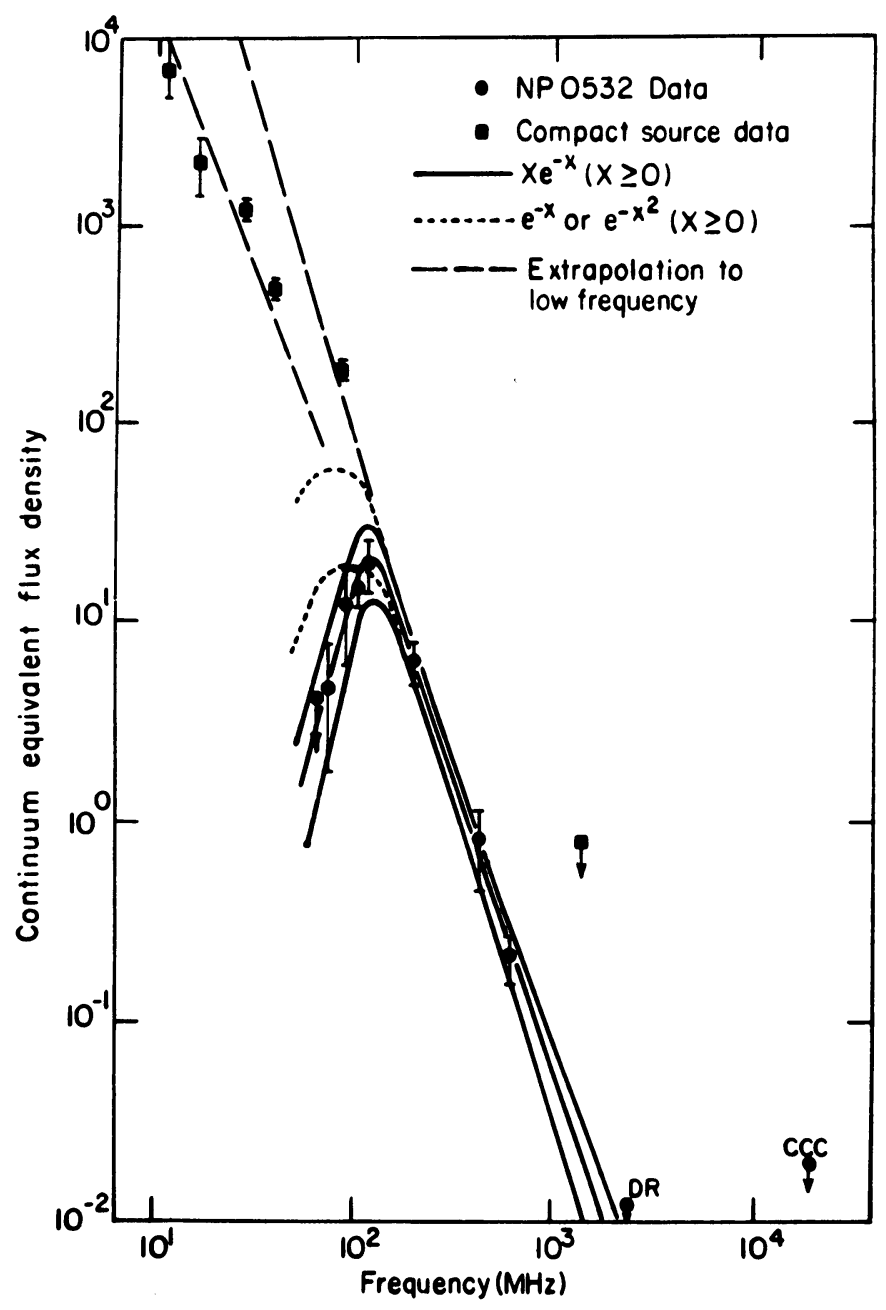

Fig. 4. The radio spectra of NP 0532 and the compact radio source in the Crab Nebula. The points for the pulsar are from Rankin et al. (1970) except for the point DR which is by Downs and Reichley, and the point CCC by Counselman. Compact source values are by Bridle. The solid curves are the spectrum predicted by the model in which interstellar broadening causes a broadening proportional to $x e^{-x}$. The parallel solid curves give the error envelope for the fit. 
at low frequencies is a continuation of the high frequency spectrum. It is very striking that the flux densities of the compact continuous source of radiation at the center of the Crab Nebula fall almost exactly on the extrapolation of the high frequency portion of the pulsar spectrum. This makes a very strong case that the compact source is the pulsar, specifically its radiation is the pulsed radiation converted to continuous radiation by the interstellar medium.

The picture of the situation leads to the additional prediction that the apparent angular size of the source will increase at the lowest frequencies where we receive radiation from ever more deviant rays at longer wavelengths. The theory gives the relation $\theta_{\text {scat }}^{2}=8 c \Delta t / R$ between $\Delta t$ and the apparent size of the source $\theta_{\text {scat }}$, where $R$ is the distance to the pulsar. At $81 \mathrm{MHz}$ this relation predicts a value of $\theta_{\text {scat }}=$ $=1.1 \times 10^{-6}$ radian. Bell and Hewish (1967) report a measurement of the apparent size of the compact source of $1 \pm 0.5 \times 10^{-6}$ rad. Similarly at $26 \mathrm{MHz}$ the relation predicts an apparent size of the source of about one second of arc and indeed Cronyn has observed an apparent size of about this value at that frequency.

There are now a number of arguments leading to the conclusion that the compact source radiation is nothing more than the pulsar radiation smeared in time so as to be continuous. These arguments are: (1) A very close proximity of the measured positions of the compact radio source and the pulsar. The difference in position is less than 15". (2) The unusually high brightness temperature computed for the compact source. The compact source has presented a serious problem in this regard and has led to suggestions that unusual physics is involved. The hypothesis that the source is the pulsar solves the problem since the physics of the pulsar can easily produce the high brightness temperatures. (3) Compatibility of the spectra of the pulsar and the compact source as discussed. (4) The consistency between the measured appearant size of the compact source as a function of radio frequency as compared to the theoretical predictions of the size to be expected from propagation effects.

A very striking characteristic of the Crab Nebula pulsar is its sporadic emission of individual very intense strong pulses at random intervals of several minutes. Approximately one in 10000 is one of these strong pulses. Individual pulses with a peak flux density of 20000 flux units have been observed by Heiles at $430 \mathrm{MHz}$; this is a flux density 20 times that of the Crab Nebula. For brief intervals, the Crab pulsar is the brightest cosmic radio source in the sky at this frequency. Figure 5 shows an example of one of these strong pulses as observed by Heiles, Rankin and Campbell. They observe strong pulses by recording digitally a $50 \mu \mathrm{sec}$ average of the radiation with such a sample being taken every $50 \mu \mathrm{sec}$. The strong pulses are not simply the tail of the statistical distribution of numbers of pulses versus pulse intensity. They form an independent component in the statistical distribution.

In every such strong pulse observed it is the main pulse which is very intense, with there being no evidence of great intensity in either the precursor or the interpulse. This shows very clearly that the pulse enhancement is not caused by any effect in the interstellar medium, since this would affect all components of the pulse equally. The evidence is clear that these individual strong main pulses, typically a hundred times 
stronger than the average pulse intensity, are created by a special physical mechanism in the pulsar itself.

The duration of the strong pulses is very short, being typically less than $100 \mu \mathrm{sec}$. In every strong pulse so far observed this has been true, except for a few instances

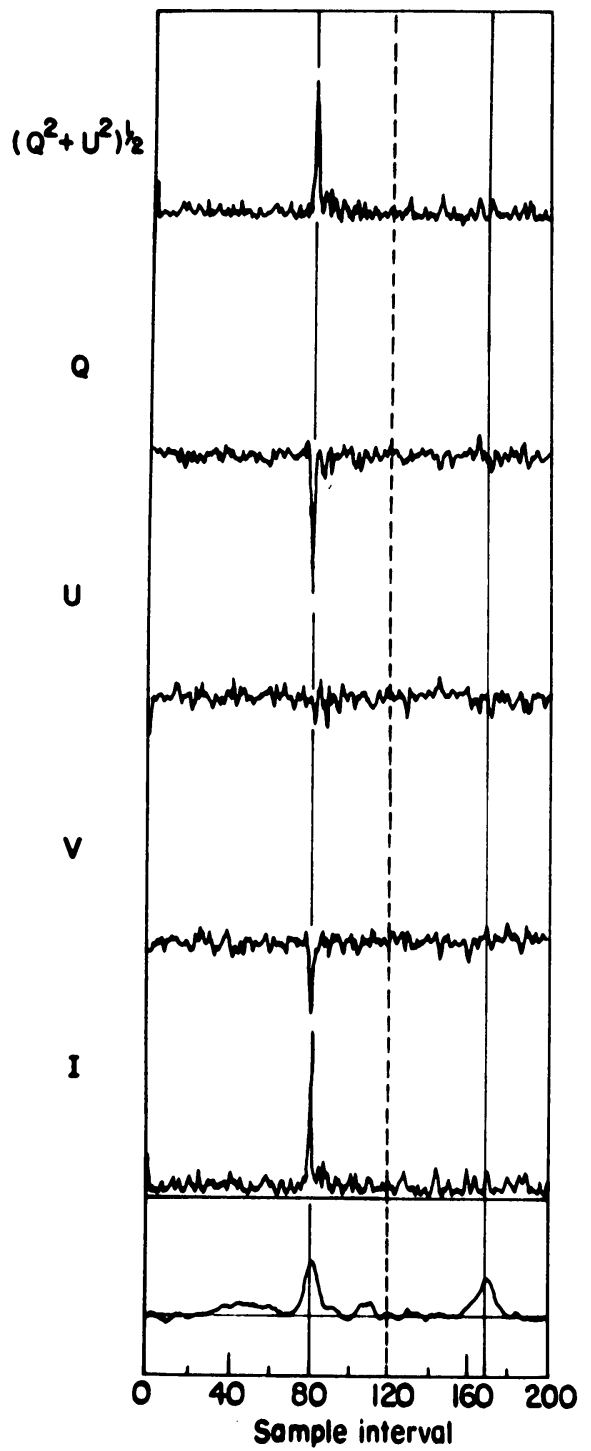

Fig. 5. Observation at $430 \mathrm{MHz}$ of four Stokes parameters of an individual strong pulse from NP 0532. The total intensity averaged over all pulses recorded on 3 November, 1969, is shown at the bottom. The sampling time is $50 \mu \mathrm{sec}$. Note that recording system ceased taking data for a time interval of 9 msec at sample interval 120. The solid vertical lines at sample interval 80 and 169 show the approximate centers of the main and interpulse, respectively. $I$ is the total intensity, $V$ is the circularly polarized Stokes parameter, and $Q$ and $U$ are the linearly polarized parameters. Note presence of strong linear and circular components. 
where a double strong pulse consisting of two such short components has been seen. The individual strong pulses show a very large degree of polarization but with no apparent systematic correlation with the pulse phase at which the strong pulse occurs. The pulse of Figure 5 is strongly elliptically polarized. The pulse polarization may be strongly linear, but at times shows strong circular polarization. There seems to be no general rule describing the polarization of the strong pulses.
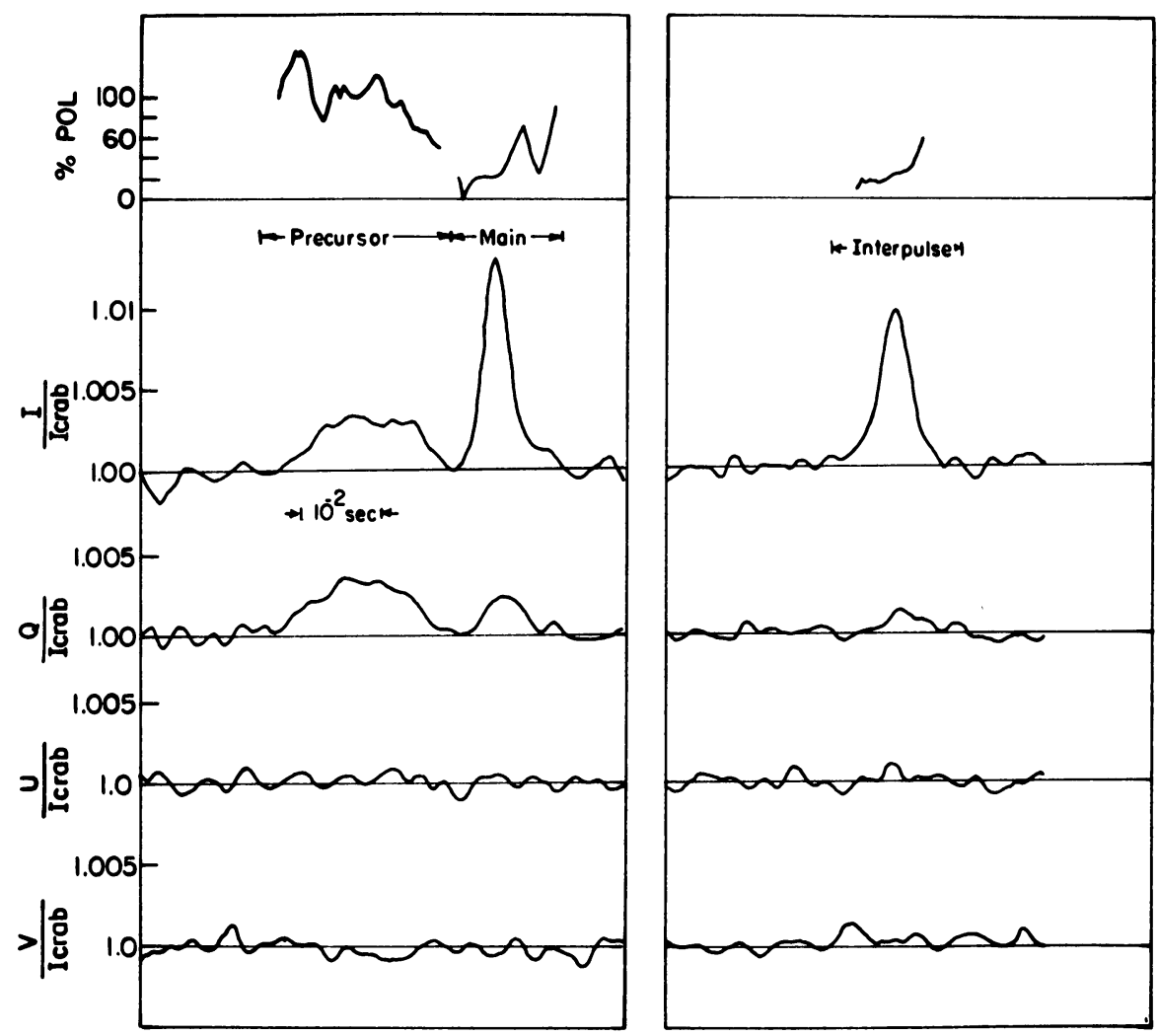

Fig. 6. The mean polarization of the Crab pulsar. The Stokes parameters $I, Q, U$ and $V$ are again given in terms of the intensity of the Crab Nebula. At the top, the percentage linear polarization is shown.

On the other hand, measurements of the average polarization seen when the mean values of Stokes parameters for tens of thousands of pulses are combined give a large systematic polarization within a pulsar pulse. Figure 6 shows this result from Arecibo by Campbell et al. (1970). As can be seen in the plots of the four Stokes parameters, there is no evidence of circular polarization whatsoever. Only one of the linearly polarized Stokes parameters shows a finite intensity. It is to be noted that the plane of linear polarization of the receiving system has been intentionally rotated so that the polarization of the Stokes parameter is parallel to the evidently persistent plane 
of polarization of pulsar signals. The results seem to show that there is no rotation of the linear polarization in the course of the pulse, contrary to the effects seen in other pulsars. The polarization of the precursor is one hundred per cent linear throughout. The linear polarization of the main pulse is about $20 \%$ and of the interpulse $13 \%$.

Accurate measurements of the time of arrival of pulses have been carried out for over a year at Arecibo primarily by Richards, Roberts and Rankin. Since these will be described in detail later, they will only be mentioned briefly here. As mentioned earlier, they have shown strong evidence that the pulsar is a rotating neutron star and that its rotational energy is being converted into the energy of relativistic particles and electromagnetic radiation in the radio, optical and X-ray regions. The short duration of the main and interpulse component at $430 \mathrm{MHz}$ have permitted extremely good timing accuracies, typically $8 \mu \mathrm{sec}$ from the data of a single day's observations. With this accuracy it has been possible to measure both the first and second derivatives in the rotation frequency of the pulsar. The first derivative leads directly to the energy released from the pulsar; the second derivative gives some evidence as to the mode by which rotational energy is converted into other forms of energy. The data on the second derivative show that the first derivative can be fairly accurately described as proportional to the rotational frequency raised to a power which is observationally in the range 2.2 to 2.4 . This power is very close to the value of three predicted by the electromagnetic dipole braking theory for pulsars. As has been pointed out by Pacini, if the plasma of the pulsar distorts the magnetic field so as to draw it out into the equatorial plane of the pulsar, we would expect an exponent less than three. The observations are consistent with this picture.

In addition to the general spindown of the pulsar, an abrupt increase in the rotational frequency was observed on 28 September 1969. This was also observed, of course, by Boynton et al. (1969) at Princeton. The decrease in pulsar period was about 100 psec, much smaller than in the similar event observed in the Vela pulsar 0833-45. Nevertheless, if this discontinuity is interpreted to be a result of a change in a moment of inertia of the neutron star it implies a release of gravitational energy of the order of $10^{41} \mathrm{erg}$, equivalent to the luminosity of the sun over a year.

In addition to the abrupt change in period, instabilities in the pulsar period with time scales of the order of days to months have been observed. Prior to the 1969 discontinuity these deviations from a secular spindown were very nearly sinusoidal in form. After the 1969 event the quasisinusoidal variation continued for several months and then was transformed into an irregular variation of the pulsar with a time scale of the order of months. As will be seen in later papers in this symposium the interpretation of the irregularities in the pulsar timing is extremely complex and challenging.

\section{References}

Bell, S. J. and Hewish, A.: 1967, Nature 213, 1214.

Boynton, P. E., Groth, E. J., III, Partridge, R. B., and Wilkinson, D. T.: 1969, IAU Circular 2179.

Campbell, D. B., Heiles, C. E., and Rankin, J. M.: 1970, Nature 225, 527. 
Cocke, W. J., Disney, M. J., and Taylor, D. J.: 1969, Nature 221, 525.

Comella, J. M., Craft, H. D., Jr., Lovelace, R. V. E., Sutton, J. M., and Tyler, G. L.: 1969, Nature 221, 453.

Gold, T.: 1969, Nature 221, 25.

Heiles, C. E., Rankin, J. M., and Campbell, D. B.: 1970, Nature 226, 529.

Rankin, J. M., Comella, J. M., Craft, H. D., Jr., Richards, D. W., Campbell, D. B., Counselman, C. C. III: 1970, Astrophys. J. $162,707$.

Richards, D. W. and Comella, J. M.: 1969, Nature 222, 551.

Salpeter, E. E.: 1969, Nature 221, 31.

Staelin, D. H. and Reifenstein, E. C., III: 1968, Science 162, 1481.

Staelin, D. H. and Sutton, J. M.: 1970, Nature 226, 69.

\section{Discussion}

$R$. C. Jennison: Are any other compact low frequency sources known which nearly coincide in position with any other pulsars?

F. D. Drake: No. Craft observed the region of all the Arecibo observable pulsars, and found no associated continuous sources with an upper limit of about $2 \%$ of the mean pulsar flux density.

J.P. Ostriker: Is it difficult to detect distant pulsars with very short periods and with large dispersion measures?

F. D. Drake: Yes, the broadening prevents the detection of short period pulsars, say with periods less than about 100 milliseconds, when the dispersion measure is more than about 100 .

J. Kristian: Do you know the absolute angle for the linear polarization of the Crab pulsar?

F. D. Drake: No, the measurement wasn't made at Arecibo.

J. E. Baldwin: Given a pulse shape at a high frequency where smoothing is small and also at a low frequency where smoothing is present, it is evidently possible to derive uniquely the smoothing function for that frequency Has this been done rather than attempting to fit algebraic functions to the data?

F. D. Drake: Counselman of MIT has attempted to fit a function at a single frequency and found that the $x e^{-x}$ broadening function gives the best fit. Note however that this broadening is non-linear in frequency and so observations at two frequencies are not enough. The models I have discussed attempt to include this frequency dependence.

$W . J$. Cocke: Bridle has measured the diameter of the compact source to be $0.2 \mathrm{arcsec}$, at around $20 \mathrm{MHz}$. Have you any comment about that? (A. H. Bridle, Nature 225, (1970) 1035.)

$F$. D. Drake: I did not know of the size limit of $0^{\prime \prime} .2$ at low radio frequencies. This value disagrees with the observations of Cronyn. 